\title{
Local gradient estimate for $p$-harmonic functions on Riemannian manifolds
}

\author{
XiaOdong Wang and Lei Zhang
}

\begin{abstract}
For positive $p$-harmonic functions on Riemannian manifolds, we derive a gradient estimate and Harnack inequality with constants depending only on the lower bound of the Ricci curvature, the dimension $n, p$ and the radius of the ball on which the function is defined. Our approach is based on a careful application of the Moser iteration technique and is different from Cheng-Yau's method [2] employed by Kostchwar and $\mathrm{Ni}$ [5], in which a gradient estimate for positive $p$-harmonic functions is derived under the assumption that the sectional curvature is bounded from below.
\end{abstract}

\section{Introduction}

The study of harmonic functions on Riemannian manifolds has been one of the central subjects in geometric analysis. In their classical work, Cheng-Yau [15] derived the following gradient estimate for positive harmonic functions on Riemannian manifolds:

Theorem A (Cheng-Yau). Let $M$ be an n-dimensional complete Riemannian manifold with Ric $\geq-(n-1) \kappa$, where $\kappa \geq 0$ is a constant. Suppose that $u$ is a positive harmonic function on a geodesic ball $B(o, R)$. Then

$$
\sup _{B(o, R / 2)} \frac{|\nabla u|}{u} \leq C_{n} \frac{1+R \sqrt{\kappa}}{R}
$$

where $C_{n}$ is a constant depending only on $n$.

An important feature of Cheng-Yau's estimate is that the RHS (which stands for the right-hand side) of (1.1) depends only on $n, k$ and $R$, it does not depend on the lower bound of the injectivity radius or a global coordinate system. From partial differential equations (PDE) viewpoints, 
deriving a Harnack inequality requires some bounds on the coefficients in some fixed coordinate system, thus not suitable for many problems defined on manifolds. We also observe that the RHS of (1.1) is optimal in the sense that even for $\kappa>0$, the bound stays bounded when $R \rightarrow \infty$.

There are two major ingredients in the proof of Theorem A. First a Bochner formula is used to derive a lower bound of the Laplacian of $|\nabla u|^{2}$ for a harmonic function $u$ in terms of the lower bound of the Ricci tensor. The second major ingredient is a clever application of the maximum principle. The trick is to multiply $|\nabla u|^{2}$ by a cut-off function, derive a new differential inequality for the product and then apply the maximum principle. The cutoff function is constructed using the distance function. As a result, the new differential inequality involves the Laplacian of the distance function. As is well known, the Riemannian distance function is uniformly Lipschitz and its Laplacian has an upper bound depending on the lower bound of the Ricci tensor.

Cheng-Yau's approach turned out to be very useful and some important results for other problems are deeply influenced by Theorem A. For example, Li [8] obtained the sharp lower bound for the first eigenvalue of a manifold, which was later generalized by Li-Yau [9]. Similar results were also obtained by Li-Yau [10] for heat equations. Cheng [1] and Choi [3] obtained gradient estimates for harmonic mappings, etc. We refer to $[14,15]$ and the more recent survey [7] for an overview of the subject.

$p$-harmonic functions are natural extensions of harmonic functions from a variational point of view. It has been extensively studied because of its various interesting features and applications. Compared with the theory for harmonic functions the study of $p$-harmonic functions is generally harder because the equation, even though elliptic, is degenerate and the regularity results are far weaker (see, for example [16]). Recently, there has been renewed interest in $p$-harmonic functions. In particular, Moser [11] established a nice connection between $p$-harmonic functions and the inverse mean curvature flow. In a recent paper [5] Kotschwar and Ni derived, among other things, a local gradient estimate for $p$-harmonic functions under the assumption that the sectional curvature is bounded from below. It is remarkable that the constant in their estimate does not blowup when $p \rightarrow 1$, which leads to interesting results on the inverse mean curvature flow problems. Their proof follows the same strategy introduced by Cheng-Yau [2] for harmonic functions (i.e., $p=2$ ). However, for general $p$-harmonic functions, the computation involves the full Hessian of the distance function when the cut-off function is introduced. As a result, a lower bound on the sectional curvature has to be assumed in [5]. 
Kotschwar and Ni speculated that their estimate may hold if only a lower bound on the Ricci tensor is assumed. The main result of this paper is to establish the following theorem:

Theorem 1.1. Let $\left(M^{n}, g\right)$ be a complete Riemannian manifold with Ric $\geq-(n-1) \kappa$. Assume that $v$ is a positive p-harmonic function on the ball $B(o, R) \subset M$. Then

$$
\frac{|\nabla v|}{v} \leq C_{p, n}(1+\sqrt{\kappa} R) / R \quad \text { on } \quad B(o, R / 2) .
$$

where $C_{p, n}$ depends only on $p$ and $n$.

The proof of Theorem 1.1 will be presented in Section 2. As far as the second major ingredient of Cheng-Yau's proof is concerned, our approach follows a different strategy by carefully using the Moser iteration technique. This approach only involves differentiating the distance function once and hence bypasses the difficulty of handling the full Hessian of the distance function. In the special case $p=2$, when $p$-harmonic function are just harmonic functions, Theorem 1.1 is exactly Cheng-Yau's theorem. An immediate consequence of Theorem 1.1 is the following Harnack inequality.

Theorem 1.2. Let $\left(M^{n}, g\right)$ be a complete Riemannian manifold with Ric $\geq-(n-1) \kappa$. Assume that $v$ is a positive p-harmonic function on the ball $B(o, R) \subset M$. Then there exists a constant $C_{p, n}$ such that for any $x, y \in B(o, R / 2)$,

$$
v(x) / v(y) \leq e^{C_{p, n}(1+\sqrt{\kappa} R)} .
$$

It follows that if $\mathrm{Ric} \geq 0$, then we have a uniform constant $c_{p, n}$ (independent of $R$ ) s.t.

$$
\sup _{B(o, R / 2)} v \leq c_{p, n} \inf _{B(o, R / 2)} v
$$

This was already proved by Rigol et al. [12]. In fact, they proved the stronger result that (1.2) holds provided that the volume is doubling and a weak Poincare inequality holds. See also [4].

Another standard application of Theorem 1.1 is the following Liouville theorem, which was also deduced from the Harnack inequality in [12].

Let $u$ be a p-harmonic function bounded from above or below on a complete Riemannian manifold with non-negative Ricci tensor, then $u$ is constant. 
Finally, we point out that our constant $C_{p, n}$ in Theorem 1.1 becomes unbounded as $p \rightarrow 1$, while in Kotschwar-Ni's result, all the constants stay bounded when $p \rightarrow 1$. We do not know if the method can be tweaked to remove this defect.

\section{The gradient estimate for the $p$-harmonic functions}

$p$-harmonic functions arise naturally as critical points of the $L^{p}(p>1)$ norm of the gradient. Let $\left(M^{n}, g\right)$ be a complete Riemannian manifold and $\Omega \subset M$ an open set. A function $v \in W_{l o c}^{1, p}(\Omega)$ is $p$-harmonic if

$$
\operatorname{div}\left(|\nabla v|^{p-2} \nabla v\right)=0
$$

in the weak sense, i.e.,

$$
\int_{U}|\nabla v|^{p-2}\langle\nabla v, \nabla \xi\rangle=0
$$

for all $\xi \in W_{0}^{1, p}(\Omega)$. By Tolksdorf [16], for example, $v$ must be $C^{1, \alpha}$. Moreover, $v \in W_{\text {loc }}^{2,2}$ if $p \geq 2 ; v \in W_{\text {loc }}^{2, p}$ if $1<p<2$. Away from $\{\nabla v=0\}, v$ is in fact smooth.

Suppose that $v$ is positive. Set $u=-(p-1) \log v$. Then $u$ satisfies

$$
\operatorname{div}\left(|\nabla u|^{p-2} \nabla u\right)=|\nabla u|^{p}
$$

Let $f=|\nabla u|^{2}$. We define

$$
\mathcal{L}(\psi)=\operatorname{div}\left(f^{p / 2-1} A(\nabla \psi)\right)-p f^{p / 2-1}\langle\nabla u, \nabla \psi\rangle
$$

where

$$
A=i d+(p-2) \frac{\nabla u \otimes \nabla u}{|\nabla u|^{2}} .
$$
lation.

We need the following lemma from [5] and the proof is by direct calcu-

\section{Lemma 2.1.}

$$
\mathcal{L}(f)=2 f^{p / 2-1}\left(\left|D^{2} u\right|^{2}+\operatorname{Ric}(\nabla u, \nabla u)\right)+\left(\frac{p}{2}-1\right)|\nabla f|^{2} f^{p / 2-2} .
$$


Remark 2.1. Lemma 2.1 holds point-wisely in $\{x: f(x)>0\}$. From the gradient estimate of [16] we know that $f=|\nabla u|^{2} \in C^{\alpha}$ for some $\alpha>0$ and $f \in W_{\text {loc }}^{1, \beta}$ for some $\beta>1$.

We choose a local orthonormal frame $\left\{e_{i}\right\}$ with $e_{1}=\nabla u /|\nabla u|$. Then (2.1) takes the following form:

$$
(p-1) u_{11}+\sum_{i=2}^{n} u_{i i}=f
$$

Therefore,

$$
\begin{aligned}
\left|D^{2} u\right|^{2} & \geq u_{11}^{2}+2 \sum_{i=2}^{n} u_{1 i}^{2}+\sum_{i=2}^{n} u_{i i}^{2} \\
& \geq u_{11}^{2}+2 \sum_{i=2}^{n} u_{1 i}^{2}+\frac{1}{n-1}\left(\sum_{i=2}^{n} u_{i i}\right)^{2} \\
& =u_{11}^{2}+2 \sum_{i=2}^{n} u_{1 i}^{2}+\frac{1}{n-1}\left(f-(p-1) u_{11}\right)^{2} \\
& =\frac{1}{n-1} f^{2}-\frac{2(p-1)}{n-1} f u_{11}+\left(1+\frac{(p-1)^{2}}{n-1}\right) u_{11}^{2}+2 \sum_{i=2}^{n} u_{1 i}^{2} \\
& \geq \frac{1}{n-1} f^{2}-\frac{2(p-1)}{n-1} f u_{11}+a_{0} \sum_{i=1}^{n} u_{1 i}^{2}
\end{aligned}
$$

where $a_{0}=1+\min \left(\frac{(p-1)^{2}}{n-1}, 1\right)>1$. Using the identities

$$
\begin{aligned}
2 f u_{11} & =\langle\nabla u, \nabla f\rangle, \\
\sum_{i=1}^{n} u_{1 i}^{2} & =\frac{1}{4} \frac{|\nabla f|^{2}}{f}
\end{aligned}
$$

we end up with

$$
\left|D^{2} u\right|^{2} \geq \frac{1}{n-1} f^{2}-\frac{(p-1)}{n-1}\langle\nabla u, \nabla f\rangle+\frac{a_{0}}{4} \frac{|\nabla f|^{2}}{f}
$$


Assume that Ric $\geq-(n-1) \kappa$. Therefore,

$$
\begin{aligned}
\mathcal{L}(f) \geq & -2(n-1) \kappa f^{p / 2}+\left(\frac{p+a_{0}}{2}-1\right)|\nabla f|^{2} f^{p / 2-2} \\
& +\frac{2}{n-1} f^{p / 2+1}-\frac{2(p-1)}{n-1} f^{p / 2-1}\langle\nabla u, \nabla f\rangle \\
\geq & -2(n-1) k f^{p / 2}+\frac{2}{n-1} f^{p / 2+1}-\frac{2(p-1)}{n-1} f^{p / 2-1}\langle\nabla u, \nabla f\rangle
\end{aligned}
$$

Equation (2.2) holds wherever $f$ is strictly positive. Let $K=\{x \in \Omega$ : $f(x)=0\}$. Then for any non-negative function $\psi$ with compact support in $\Omega \backslash K$, we have

$$
\begin{aligned}
& \int_{\Omega}\left\langle f^{p / 2-1} \nabla f+(p-2) f^{p / 2-2}\langle\nabla u, \nabla f\rangle \nabla u, \nabla \psi\right\rangle \\
& \quad+p \int_{\Omega} f^{p / 2-1}\langle\nabla u, \nabla f\rangle \psi+\frac{2}{n-1} \int_{\Omega} f^{p / 2+1} \psi \\
& \quad \leq 2(n-1) k \int_{\Omega} f^{p / 2} \psi+\frac{2(p-1)}{n-1} \int_{\Omega} f^{p / 2-1}\langle\nabla u, \nabla f\rangle \psi
\end{aligned}
$$

In particular, let $\epsilon>0$ and $\psi=f_{\epsilon}^{b} \eta^{2}$ where $f_{\epsilon}=(f-\epsilon)^{+}, \eta \in C_{0}^{\infty}\left(B_{R}\right)$ is non-negative, $b>1$ is to be determined later. Then direct computation yields

$$
\nabla \psi=b f_{\epsilon}^{b-1} \nabla f \eta^{2}+2 f_{\epsilon}^{b} \eta \nabla \eta
$$

Using the above in (2.3) we obtain

$$
\begin{aligned}
b \int_{B_{R}} & \left(f^{p / 2-1} f_{\epsilon}^{b-1}|\nabla f|^{2}+(p-2) f^{p / 2-2}\langle\nabla u, \nabla f\rangle^{2} f_{\epsilon}^{b-1}\right) \eta^{2} \\
& +2(p-2) \int_{B_{R}} f^{p / 2-2}\langle\nabla u, \nabla f\rangle f_{\epsilon}^{b} \eta(\nabla u, \nabla \eta) \\
& +2 \int_{\Omega} f^{p / 2-1} f_{\epsilon}^{b} \eta\langle\nabla f, \nabla \eta\rangle \\
& +p \int_{\Omega} f^{p / 2-1}\langle\nabla u, \nabla f\rangle f_{\epsilon}^{b} \eta^{2}+\frac{2}{n-1} \int_{\Omega} f^{p / 2+1} f_{\epsilon}^{b} \eta^{2} \\
& \leq 2(n-1) \kappa \int_{\Omega} f^{p / 2} f_{\epsilon}^{b} \eta^{2}+\frac{2(p-1)}{n-1} \int_{\Omega} f^{p / 2-1}\langle\nabla u, \nabla f\rangle f_{\epsilon}^{b} \eta^{2}
\end{aligned}
$$


Since $u \in C^{1, \alpha}, f \in C^{\alpha}$ and $\nabla f \in L^{\beta}(\Omega)$ for some $\alpha>0$ and $\beta>1$, we see that except for the first term, all the other terms converge to the corresponding form without $\epsilon$. For the first term, observe that

$$
f^{p / 2-1} f_{\epsilon}^{b-1}|\nabla f|^{2}+(p-2) f^{p / 2-2}\langle\nabla u, \nabla f\rangle^{2} f_{\epsilon}^{b-1} \geq a_{1} f^{p / 2-1} f_{\epsilon}^{b-1}|\nabla f|^{2}
$$

where $a_{1}=1$ if $p \geq 2$ and $a_{1}=(p-1)$ if $p \in(1,2)$. Thus by passing $\epsilon$ to 0 we have

$$
\begin{aligned}
& b a_{1} \int_{\Omega} f^{p / 2+b-2}|\nabla f|^{2} \eta^{2} \\
& \quad+2(p-2) \int_{\Omega} f^{\frac{p-4}{2}+b}\langle\nabla u, \nabla f\rangle \eta\langle\nabla u, \nabla \eta\rangle+2 \int_{\Omega} f^{\frac{p-2}{2}+b} \eta\langle\nabla f, \nabla \eta\rangle \\
& \quad+p \int_{\Omega} f^{\frac{p-2}{2}+b}\langle\nabla u, \nabla f\rangle \eta^{2}+\frac{2}{n-1} \int_{\Omega} f^{\frac{p+2}{2}+b} \eta^{2} \\
& \quad \leq 2(n-1) k \int_{\Omega} f^{\frac{p}{2}+b} \eta^{2}+\frac{2(p-1)}{n-1} \int_{\Omega} f^{\frac{p-2}{2}+b}\langle\nabla u, \nabla f\rangle \eta^{2}
\end{aligned}
$$

From now on we use $a_{1}, a_{2}, \ldots$, etc. to denote constants depending only on $p$ and $n$. Combining terms in (2.5) using the definition of $f$ we have

$$
\begin{aligned}
& a_{1} b \int_{\Omega} f^{p / 2+b-2}|\nabla f|^{2} \eta^{2}+\frac{2}{n-1} \int_{\Omega} f^{p / 2+1+b} \eta^{2} \\
& \leq 2(n-1) \kappa \int_{\Omega} f^{p / 2+b} \eta^{2}+a_{2} \int_{\Omega} f^{\frac{p-1}{2}+b}|\nabla f| \eta^{2} \\
& \quad+a_{3} \int_{\Omega} f^{p / 2+b-1}|\nabla f||\nabla \eta| \eta
\end{aligned}
$$

For $R_{3}$ (the third term on the RHS, $L_{1}, L_{2}, R_{1}$ etc. are understood similarly) in (2.6) we have

$$
\left|R_{3}\right| \leq \frac{a_{1} b}{4} \int_{\Omega} f^{b+p / 2-2}|\nabla f|^{2} \eta^{2}+\frac{a_{4}}{b} \int_{\Omega}|\nabla \eta|^{2} f^{b+p / 2}
$$

Also by Cauchy's inequality $R_{2}$ of $(2.6)$ can be estimated as

$$
\left|R_{2}\right| \leq \frac{a_{1} b}{4} \int_{\Omega} f^{p / 2+b-2}|\nabla f|^{2} \eta^{2}+\frac{a_{5}}{b} \int_{\Omega} f^{b+p / 2+1} \eta^{2} .
$$


With these two inequalities we obtain

$$
\begin{aligned}
& \frac{a_{1} b}{2} \int_{\Omega} f^{p / 2+b-2}|\nabla f|^{2} \eta^{2}+\frac{2}{n-1} \int_{\Omega} f^{p / 2+1+b} \eta^{2} \\
& \quad \leq \int_{\Omega}\left(2(n-1) \kappa \eta^{2}+\frac{a_{4}}{b}|\nabla \eta|^{2}\right) f^{p / 2+b}+\frac{a_{5}}{b} \int_{\Omega} f^{b+p / 2+1} \eta^{2}
\end{aligned}
$$

By requiring

$$
\frac{a_{5}}{b}<\frac{1}{n-1}
$$

we see that the last term in on the RHS of (2.7) is majorized by the last term on the LHS. Therefore, we have

$$
\begin{aligned}
& \frac{a_{1} b}{2} \int_{\Omega} f^{p / 2+b-2}|\nabla f|^{2} \eta^{2}+\frac{1}{n-1} \int_{\Omega} f^{p / 2+1+b} \eta^{2} \\
& \quad \leq 2(n-1) \kappa \int_{\Omega} f^{p / 2+b} \eta^{2}+\frac{a_{4}}{b} \int_{\Omega}|\nabla \eta|^{2} f^{p / 2+b}
\end{aligned}
$$

For the first term on the LHS we use

$$
\left|\nabla\left(f^{p / 4+b / 2} \eta\right)\right|^{2} \leq \frac{1}{2}\left(\frac{p}{2}+b\right)^{2} f^{p / 2+b-2}|\nabla f|^{2} \eta^{2}+2 f^{p / 2+b}|\nabla \eta|^{2}
$$

From the above we obtain

$$
\begin{aligned}
& \int_{\Omega}\left|\nabla\left(f^{p / 4+b / 2} \eta\right)\right|^{2}+d_{1} \int_{\Omega} f^{p / 2+1+b} \eta^{2} \\
& \leq \kappa d_{2} \int_{\Omega} f^{p / 2+b} \eta^{2}+a_{7} \int_{\Omega}|\nabla \eta|^{2} f^{b+p / 2}
\end{aligned}
$$

where $d_{1} \sim b, d_{2} \sim b$ (recall $b>1, d_{1} \sim b$ means $d_{1}$ is comparable to $b, d_{2} \sim b$ is understood the same way).

The following Sobolev embedding theorem of Saloff-Coste plays an important role in our approach:

Theorem B (Theorem 3.1 of [13]). Let $\left(M^{n}, g\right)$ be a complete Riemannian manifold with Ric $\geq-(n-1) \kappa$. For $n>2$, there exists $C$, depending only on $n$, such that for all $B \subset M$ of radius $R$ and volume $V$ we 
have for $f \in C_{0}^{\infty}(B)$

$$
\left(\int|f|^{2 q}\right)^{1 / q} \leq e^{C(1+\sqrt{\kappa} R)} V^{-2 / n} R^{2}\left(\int|\nabla f|^{2}+R^{-2} f^{2}\right)
$$

where $q=n /(n-2)$. For $n=2$, the above inequality holds with $n$ replaced by any fixed $n^{\prime}>2$.

From now on, we assume $\Omega=B(o, R)$. Theorem $\mathrm{B}$ gives

$$
\begin{aligned}
& \left(\int_{\Omega} f^{\frac{n(p / 2+b)}{n-2}} \eta^{\frac{2 n}{n-2}}\right)^{(n-2) / n} \\
& \quad \leq e^{c_{0}(1+\sqrt{\kappa} R)} V^{-\frac{2}{n}}\left(R^{2} \int_{\Omega}\left|\nabla\left(f^{p / 4+b / 2} \eta\right)\right|^{2}+\int_{\Omega} f^{p / 2+b} \eta^{2}\right) .
\end{aligned}
$$

where $c_{0}(n, p)>0$ depends only on $n, p$. Let $b_{0}=c_{1}(n, p)(1+\sqrt{\kappa} R)$ with $c_{1}(n, p)$ large enough to make $b_{0}$ satisfy (2.8), then (2.9) and (2.10) combined gives

$$
\begin{aligned}
& \left(\int_{\Omega} f^{\frac{n(p / 2+b)}{n-2}} \eta^{\frac{2 n}{n-2}}\right)^{(n-2) / n}+a_{8} b R^{2} e^{c_{2} b_{0}} V^{-2 / n} \int_{\Omega} f^{p / 2+1+b} \eta^{2} \\
& \quad \leq a_{9} b_{0}^{2} b e^{c_{2} b_{0}} V^{-2 / n} \int_{\Omega} f^{p / 2+b} \eta^{2}+a_{10} e^{c_{2} b_{0}} V^{-2 / n} R^{2} \int_{\Omega}|\nabla \eta|^{2} f^{p / 2+b} .
\end{aligned}
$$

Lemma 2.2. Let $b_{1}=\left(b_{0}+\frac{p}{2}\right) \frac{n}{n-2}$. Then there exists $c_{3}(n, p)>0$ such that

$$
\|f\|_{L^{b_{1}}\left(B_{3 R / 4}\right)} \leq c_{3} \frac{b_{0}^{2}}{R^{2}} V^{1 / b_{1}}
$$

Proof of Lemma 2.2. Let $b=b_{0}$ in (2.11), then by comparing $L_{2}$ and $R_{1}$ of (2.11) we observe that

$$
a_{9} b_{0}^{3} f^{p / 2+b_{0}}<\frac{1}{2} a_{8} b_{0} R^{2} f^{p / 2+1+b_{0}}
$$

if $f>a_{11} b_{0}^{2} R^{-2}$. Thus in the evaluation of $R_{1}$ we decompose $\Omega$ into two subregions, one over the places where $f \leq a_{11} b_{0}^{2} R^{-2}$ and the second region 
is the complement of the first region. With this decomposition we have

$$
R_{1} \leq a_{12}^{b_{0}} b_{0}^{3}\left(\frac{b_{0}}{R}\right)^{p+2 b_{0}} e^{c_{2} b_{0}} V^{1-2 / n}+\frac{L_{2}}{2} .
$$

Now (2.11) with $b=b_{0}$ can be written as

$$
\begin{aligned}
& \left(\int_{\Omega} f^{\frac{n\left(p / 2+b_{0}\right)}{n-2}} \eta^{\frac{2 n}{n-2}}\right)^{(n-2) / n}+\frac{a_{8}}{2} b_{0} R^{2} e^{c_{2} b_{0}} V^{-2 / n} \int_{\Omega} f^{p / 2+1+b_{0}} \eta^{2} \\
& \leq a_{12}^{b_{0}} b_{0}^{3}\left(\frac{b_{0}}{R}\right)^{p+2 b_{0}} e^{c_{2} b_{0}} V^{1-2 / n}+a_{10} e^{c_{2} b_{0}} V^{-2 / n} R^{2} \int_{\Omega}|\nabla \eta|^{2} f^{p / 2+b_{0}}
\end{aligned}
$$

Now we choose $\eta$ to make $R_{2}$ in (2.13) dominated by the LHS. Let $\eta_{1} \in C_{0}^{\infty}\left(B_{R}\right)$ satisfy

$$
0 \leq \eta_{1} \leq 1, \quad \eta_{1} \equiv 1 \quad \text { in } B_{3 R / 4}, \quad\left|\nabla \eta_{1}\right| \leq C(n) / R
$$

Let $\eta=\eta_{1}^{m}$ where $m=b_{0}+\frac{p}{2}+1$. Direct computation shows

$$
R^{2}|\nabla \eta|^{2} \leq a_{13} b_{0}^{2} \eta^{\frac{2 b_{0}+p}{b_{0}+p / 2+1}} .
$$

By (2.14) and Young's inequality, the $R_{2}$ of (2.13) can be written as

$$
\begin{aligned}
a_{10} & R^{2} \int|\nabla \eta|^{2} f^{b_{0}+p / 2} \\
& \leq a_{14} b_{0}^{2} \int_{\Omega} f^{b_{0}+p / 2} \eta^{\frac{2 b_{0}+p}{b_{0}+p / 2+1}} \\
& \leq a_{14} b_{0}^{2}\left(\int_{\Omega} f^{b_{0}+p / 2+1} \eta^{2}\right)^{\frac{b_{0}+p / 2}{b_{0}+p / 2+1}} V^{\frac{1}{b_{0}+p / 2+1}} \\
& \leq \frac{a_{8} b_{0}}{2} R^{2} \int_{\Omega} f^{b_{0}+p / 2+1} \eta^{2}+a_{15}^{b_{0}+p / 2} \frac{b_{0}^{b_{0}+p / 2+2}}{R^{2 b_{0}+p}} V .
\end{aligned}
$$

With the estimates on $R_{1}, R_{2}$ we arrive at

$$
\left(\int_{B_{3 R / 4}} f^{\left(b_{0}+p / 2\right) n /(n-2)}\right)^{(n-2) / n} \leq a_{16}^{b_{0}} e^{c_{2} b_{0}} V^{1-2 / n} b_{0}^{3}\left(\frac{b_{0}}{R}\right)^{p+2 b_{0}}
$$

Recall $b_{1}=\left(b_{0}+\frac{p}{2}\right) \frac{n}{n-2}$. Taking the $1 /\left(b_{0}+p\right)$ root on both sides of (2.15) we have

$$
\|f\|_{L^{b_{1}}\left(B_{3 R / 4}\right)} \leq a_{17} V^{\frac{1}{b_{1}}} b_{0}^{2} / R^{2} .
$$


Lemma 2.2 is established.

Now we go back to $(2.11)$, by ignoring $L_{2}$ we have

$$
\begin{aligned}
& \left(\int_{\Omega} f^{\frac{n(p / 2+b)}{n-2}} \eta^{\frac{2 n}{n-2}}\right)^{(n-2) / n} \\
& \quad \leq a_{17} \frac{e^{c_{2} b_{0}}}{V^{2 / n}} \int_{\Omega}\left(\left(b_{0}^{2} b \eta^{2}+R^{2}|\nabla \eta|^{2}\right) f^{b+p / 2} .\right.
\end{aligned}
$$

To apply the Moser iteration we set

$$
b_{l+1}=b_{l} \frac{n}{n-2}, \quad \Omega_{l}=B\left(o, \frac{R}{2}+\frac{R}{4^{l}}\right), \quad l=1,2, \ldots,
$$

and choose $\eta_{l} \in C_{0}^{\infty}(\Omega)$ s.t.

$$
\eta_{l} \equiv 1 \text { in } \Omega_{l+1}, \quad \eta_{l} \equiv 0 \text { in } \Omega \backslash \Omega_{l}, \quad\left|\nabla \eta_{l}\right| \leq \frac{C 4^{l}}{R}, \quad 0 \leq \eta_{l} \leq 1 .
$$

Then in (2.16), by letting $b+\frac{p}{2}=b_{l}, \eta=\eta_{l}$ we have

$$
\left(\int_{\Omega_{l+1}} f^{b_{l+1}}\right)^{\frac{1}{b_{l+1}}} \leq\left(a_{17} \frac{e^{c_{2} b_{0}}}{V^{\frac{2}{n}}}\right)^{\frac{1}{b_{l}}}\left(\int_{\Omega_{l}}\left(b_{0}^{2} b_{l}+R^{2}\left|\nabla \eta_{l}\right|^{2}\right) f^{b_{l}}\right)^{\frac{1}{b_{l}}} .
$$

By the estimate of $\left|\nabla \eta_{l}\right|$

$$
\|f\|_{L^{b_{l+1}}\left(\Omega_{l+1}\right)} \leq\left(a_{17} \frac{e^{c_{2} b_{0}}}{V^{2 / n}}\right)^{1 / b_{l}}\left(b_{0}^{2} b_{l}+16^{l}\right)^{1 / b_{l}}\|f\|_{L^{b_{l}}\left(\Omega_{l}\right)} .
$$

Notice that $\sum_{l=1}^{\infty} \frac{1}{b_{l}}=\frac{n}{2 b_{1}}$, then (2.17) leads to

(2.18) $\|f\|_{L^{\infty}\left(B_{R / 2}\right)}$

$$
\begin{aligned}
& \leq\left(a_{18} \frac{e^{c_{2} b_{0}}}{V^{2 / n}}\right)^{\sum_{l=1}^{\infty} 1 / b_{l}} \prod_{l=1}^{\infty}\left(b_{0}^{3}\left(\frac{n}{n-2}\right)^{l}+16^{l}\right)^{1 / b_{l}}\|f\|_{L^{b_{1}\left(B_{3 R / 4}\right)}} \\
& \leq a_{19} \frac{e^{\frac{n c_{2} b_{0}}{2 b_{1}}}}{V^{1 / b_{1}}} b_{0}^{\frac{3 n}{2 b_{1}}}\|f\|_{L^{b_{1}\left(B_{3 R / 4}\right)}} .
\end{aligned}
$$

Using Lemma 2.2 in (2.18) we obtain

$$
\|f\|_{L^{\infty}\left(B_{R / 2}\right)} \leq a_{20} b_{0}^{2} / R^{2} .
$$

Thus Theorem 1.1 is established. 


\section{Acknowledgments}

Wang acknowledges support from NSF grant DMS-0905904. Zhang is supported in part by NSF grant 0900864(1027628).

\section{References}

[1] S.Y. Cheng, Liouville theorem for harmonic maps, in 'Geometry of the Laplace operator', Proc. Sympos. Pure Math., Univ. Hawaii, Honolulu, Hawaii, 1979, Proc. Sympos. Pure Math. XXXVI, Amer. Math. Soc., Providence, RI, 1980, 147-151.

[2] S.Y. Cheng and S.T. Yau, Differential equations on Riemannian manifolds and their geometric applications, Comm. Pure Appl. Math. 28 (1975), 333-354.

[3] H.I. Choi, On the Liouville theorem for harmonic maps, Proc. Amer. Math. Soc. 85(1) (1982), 91-94.

[4] I. Holopainen, Volume growth, Green's function, and parobolicity of ends, Duke Math. J. 97 (1999), 319-346.

[5] B. Kotschwar and L. Ni, Local gradient estimates of p-harmonic functions, 1/H-flow, and an entropy formula, Ann. Sci. Éc. Norm. Supér. (4) 42(1) (2009), 1-36.

[6] O.A. Ladyzhenskaya and N. Ural'tseva, Linear and quasilinear elliptic equations, Translated from the Russian by Scripta Technica, Inc. Translation editor: Leon Ehrenpreis Academic Press, New York-London, 1968, xviii +495 pp.

[7] P. Li, Harmonic functions and applications to complete manifolds, Lecture Notes (available on the author's homepage).

[8] P. Li, A lower bound for the first eigenvalue of the Laplacian on a compact manifold, Indiana Univ. Math. J. 28(6) (1979), 1013-1019.

[9] P. Li and S.T. Yau, Estimates of eigenvalues of a compact Riemannian manifold, in 'Geometry of the Laplace operator', Proc. Sympos. Pure Math., Univ. Hawaii, Honolulu, Hawaii, 1979, Proc. Sympos. Pure Math. XXXVI, Amer. Math. Soc., Providence, RI, 1980, 205-239.

[10] P. Li and S.T. Yau, On the parabolic kernel of the Schrödinger operator, Acta Math. 156(3-4) (1986), 153-201. 
[11] R. Moser, The inverse mean curvature flow and p-harmonic functions, J. Eur. Math. Soc. 9 (2007), 77-83.

[12] M. Rigoli, M. Salvatori and M. Vignati, A note on p-subharmonic functions on complete manifolds, Manuscripta Math. 92 (1997), 339-359.

[13] L. Saloff-Coste, Uniformly elliptic operators on Riemannian manifolds, J. Differential Geom. 36(2) (1992), 417-450.

[14] R. Schoen, The effect of curvature on the behavior of harmonic functions and mappings, in 'Nonlinear partial differential equations in differential geometry (Park City, UT, 1992)', IAS/Park City Math. Ser. 2, Amer. Math. Soc., Providence, RI, 1996, 127-184.

[15] R. Schoen and S.T. Yau, Lectures on differential geometry, International Press, Boston, 1994.

[16] P. Tolksdorf, Regularity for a more general class of quasilinear elliptic equations, J. Differential Equations 51(1) (1984), 126-150.

[17] S.T. Yau, Harmonic functions on complete Riemannian manifolds, Comm. Pure Appl. Math. 28 (1975), 201-228.

Department of Mathematics

Michigan State University

EAST LANSING

MI 48824

USA

E-mail address: xwang@math.msu.edu

Department of Mathematics

UNIVERSITY OF FLORIDA

358 Little Hall,

P.O. Box 118105

Gainesville

FL 32611-8105

USA

E-mail address: leizhang@ufl.edu

Received OCtober 13, 2010 
